\title{
Inhomogeneous Analysis of Novel Model of CFAR Approaches to Detect Two-Degrees of Freedom Partially-Correlated $\chi^{2}$-Targets
}

\author{
MOHAMED BAKRY EL-MASHADE \\ Electrical Engineering Dept., Faculty of Engineering, \\ Al Azhar University, Nasr City, Cairo, EGYPT
}

\begin{abstract}
Reliable and high performance radar systems have ubiquitous demand. The operation of such systems is affected by the presence of natural and artificial noise sources. One of the basic radar concepts is to decide whether the target is present or not. Meanwhile, the general objective of all radar detection schemes is to ensure that false alarms don't fluctuate randomly. Thus, to cope with an inhomogeneous changing clutter environment, it is beneficial to be able to detect both high- and low-fidelity targets while maintaining the rate of false alarm fixed. This calls for an adaptive thresholding strategy that vary the detection threshold as a function of the sensed environment, and most modern radars implement this approach automatically. The feature of constant false alarm rate (CFAR) activates the threshold in such a way that it becomes adaptive to the local clutter environment. Many alternatives have been proposed to achieve such demanded property. Owing to the diversity of the radar search environment (target multiplicity \& clutter edges), there exists no universal CFAR procedure. This prompts the necessity to investigate the composite architecture as a novel strategy. The goal of this paper is to analyze the fusion of CA, OS, and TM processors in post-detection integration of M-pulses. The primary and outlying targets are assumed to obey $\chi^{2}$-distribution with two-degrees of freedom in their fluctuation. Closed-form expression is derived for the detection performance. Our simulation results show robust behavior of the new model in the absence as well as in the presence of outlying targets. In addition, a significant improvement of the detection performance of novel strategy over the individual CFAR detectors is noticed. Moreover, the outweighing, over Neyman-Pearson (N-P) detector, of the fusion model, in ideal background, is evidently demonstrated. This ability to obtain improved performance compared to existing models is the major contribution of this work.
\end{abstract}

Keywords: CFAR techniques, non-coherent integration, clutter and interference, fluctuating targets, partiallycorrelated $\chi^{2}$-targets, target-multiplicity environments.

Received: October 3, 2020. Revised: February 25, 2021. Accepted: March 9, 2021. Published: March 16, 2021.

\section{Introduction}

Radar, with its obvious military and civilian applications, represents the cornerstone of modern integrated air defense systems [1]. The development of such technology has continued unabated to the present day. In this crucial system, the detection capability represents one of its most significant factors. In this regard, standard radar detection strategy assumes that the interference level is known and constant. This assumption allows accurate setting of a threshold that guarantees a desired level of false alarm [2]. Practically, however, interference levels are often varied. As the false alarm rate degrades due to the incidence of fading and interference, the adaptive strategy is one of the techniques that can lessen this ill effect. Therefore, constant false alarm rate (CFAR) procedures are initiated to keep the amount of false alarm under supervision in a diverse background of interference. In other words, the CFAR scenario is the main topic of the radar system designer in order to achieve a predictable and consistent performance. To attain this objective, the actual interference power must be estimated from the available data in real time, so that the detector threshold can be updated to follow the level of interference in such a way that the required false alarm rate can be safeguarded [3]. Thus, CFAR technology of detection is ought to be considered if the output data is fed directly to an automatic data processor. In such structure of adaptive detection, the choice of the proper threshold level is a compromise between missing targets and obtaining false alarms, and depends on how important it is to avoid either of them. As a consequence of this processing, the framework of CFAR is predicted to provide detection and false alarm of satisfied levels in realistic interference scenarios $[4,5]$.

Several alternatives of the CFAR scenarios have been proposed to deal with different situations in radar applications. The classical ones of these scenarios include cell-averaging (CA), order-statistics (OS), and many derived versions which are considered as combinations of them [6-12]. These procedures have 
been developed with the goal of increasing target detection probability under different operating circumstances. However, it is shown that most of the alternatives exhibit problems when facing certain situations that may appear in real environments and there is no universal CFAR technique that deals with diverse noise environments. This prompts the necessity to investigate composite adaptive procedure. Consequently, it is necessary to efficiently combine multiple effective features together. El-Mashade evaluates the performance of a pair of CFAR combinations, CA_TM and CA_OS, in heterogeneous situation $[13,14]$. On the other hand, fusion of particular decisions of the single CFAR detectors by appropriate fusion rules has been recommended [1519]. Multiple features fusion is an effective approach to target detection. With high probability of detection and low false alarms, an approach, based on parallel execution of CA, GO and SO algorithms and a fusion center based on a neural network with different fusion rules, is provided as a solution to the problem of selecting the appropriate algorithm for target detection in background clutter [16]. Recently, a new model of the fusion CFAR detector has been introduced [17]. However, it is a great challenge to design the rule of fusing different CFAR features. In this vein, ElMashade analyzed the non-homogeneous performance of this new fusion model for single pulse operation [20] as well as in multi-pulse situation [21]. Focusing on the problem of fluctuating target detection in inhomogeneous background, a novel detection method based on the fusion of multiple channels data is presented in this paper.

The target models and background environments are crucial factors in determining the radar systems detection performance. In automatic detection, received signals might fade due to target fluctuations. The availability of the statistical characteristics of a target's radar cross-section (RCS) can significantly enhance the behavior of target detection procedures. In other words, the more we know about the statistics of the target signal, the better the detection is. For this purpose, Swerling introduced five models (SWISWV), to describe the RCS statistical properties of the objects based on $\chi^{2}$-distribution of varying degrees of freedom. In time diversity systems, the detection probability is shown to be sensitive to the degree of correlation among the target echoes. According to Swerling's models, if only one pulse per scan hits a target, it is not possible to distinguish between cases I and II and cases III and IV. However, if multiple pulses are transmitted per antenna scan, the problem of detecting slow fluctuating targets (SWI \& SWIII) and fast fluctuating targets (SWII \& SWIV) can be easily overcome. Nevertheless, we should take into consideration the partial-correlation of the target signals; otherwise the processor fails to predict the actual system performance [5, 11-13].

This paper evaluates the closed-form performance expressions of an algorithm of parallel operation of $\mathrm{CA}, \mathrm{OS}$ and TM detectors with some data fusion rules that provides better final decision than single conventional CA, OS or TM algorithm in multipletarget situations. The primary and the secondary interfering targets are assumed to be fluctuating following $\chi^{2}$-distribution with two degrees of freedom in their fluctuation, when the radar receiver is supplied with a non-coherent integrator of M-pulses. A comparison of these schemes is also portrayed. In addition, partially-correlated targets performance is investigated. The paper proceeds as follows. Section 2 formulates the considered system of detection as well as the underlying assumptions needed in this study. The target multiplicity performance of the tested methodology along with its basic variants is analyzed in section 3. Several numerical simulations are provided in section 4 to evaluate the accuracy of the theoretical derivation and substantiate the effectiveness of the proposed schemes. Finally, some useful conclusions are drawn in section 5 .

\section{System Model and Theoretical Background}

In the radar receiver, the typically received, via the antenna, echoes are amplified and then passed through a detector circuitry to extract the envelope of the received signal. The resulting signal is proportional to the power of the echo and comprises of useful echo accompanied by unwanted power raised by internal receiver noise, external clutter and interference. The role of the detection circuitry is to establish the power threshold above which any echo can be probably considered to be originated from a target. In this regard, more echoes can be detected simultaneously with increasing the number of false alarms if this threshold is too low. On the contrary, if this threshold is too high, few echoes will be detected in conjunction with decreasing the number of false alarms. Generally, most detectors set the threshold on the purpose of getting a demanded rate of false alarm. If the background, against which targets are to be detected, is spatially and temporally stationary, then a fixed threshold level can be established to provide a specified probability of false alarm $\left(\mathrm{P}_{\mathrm{fa}}\right)$, controlled by the probability density function (PDF) of the noise, which is usually assumed to be Gaussian. In most fielded systems, clutter and interference sources are non-stationary and thus, the noise level varies. In this case, an adaptive threshold detector, which has a feature that automatically adjusts 
its sensitivity in accordance with the variation of interference power, must be provided. In other words, in face of the strength variation of the background level, the threshold must be altered in order to maintain the false alarm at its pre-set rate. A detector with this feature is termed as constant false alarm rate (CFAR) scheme. Thus, CFAR strategy of detection is an attractive approach that must be applied if the output data is fed directly to the automatic data processor. The threshold in this type of signal detection is set adaptively on the basis of estimated noise power.

Practically, for obtaining a predictable and consistent performance, the CFAR methodology is the main object of the radar system designer. The CFAR procedure is digitally realized. For the CFAR circuit to be efficient, it must fulfill some features. The more interesting ones include accurate fitting of the detection threshold to the clutter schema, low CFAR loss, masking avoidance of closely spaced targets, and establishing a threshold that allows point as well as extended targets to pass. Whatever the mechanism of the CFAR variant is, the structure of sliding window is regarded as its fundamental form. As Fig.(1) depicts, this window moves throughout the coverage area, and includes a group of reference cells (RC's) around a central cell, which is termed as cell under test (CUT). In this regard, the CUT is the cell for which the presence or absence or a target is to be decided. To mitigate self-interference in a real target echo situation, some guard cells (GC's) are introduced. These cells act as buffer between CUT and the training cells. To be sure that the CUT doesn't affect the threshold evaluation, its GC's are excluded from the background computation. The presence of a target in the CUT is declared if its power is greater than both the power of guard cells and the estimated power level. There is a chance for each resolution cell to occupy the position of the central cell. The RC's that have not yet occupied the center organize the trailing subset; whilst those that have been already processed constitute the leading subset. The choice of the sliding window size relies on rough knowledge of the typical clutter background. Generally, the window length $(\mathrm{N})$ should be as large as possible for the estimation process to be achieved in a good modality. Meanwhile, the size $(\mathrm{N})$ is preferred to be compatible with the typical range extension of homogeneous clutter zones in order to satisfy the statistical request of identically distributed random variables. The typical value of $\mathrm{N}$ is within the range of 16-32 cells in air traffic control radars [12].

The presence of an object is decided with the aid of the detection threshold which is the product of the estimated noise power $(Z)$ by a constant factor $(T)$. The scaling factor $\mathrm{T}$ is introduced to set a desired false alarm rate, and is evaluated according to statistical distribution of noise environment. It is of significance to note that increasing the value of $T$ reduces the false alarm rate, however, it leads to a loss in the detection level. The final decision is taken after comparing the content of CUT with the detection threshold, as illustrated in Fig.(1). The procedure will recommend that the signal belongs to a target, if the magnitude of the CUT outweighs the calculated threshold.

In order to achieve the detection performance analysis of an adaptive scheme in inhomogeneous background noise, it is assumed that the square-law detected output for any range cell $\left(\mathrm{y}_{0}\right)$ has an exponential distribution with parameter $\delta$. Thus, the resulting PDF has a form given by:

$$
f_{y_{0}}(y)=\frac{1}{\delta} e^{-y / \delta} U(y)
$$

$\mathrm{U}(\mathrm{y})$ stands for unit-step function with argument $\mathrm{y}$. Based on the operating situation, the value of the parameter $\delta$ can be assigned as:

$$
\delta \triangleq\left\{\begin{array}{ccc}
\psi & \text { for } & \text { Clear Background } \\
\psi(1+S) & \text { for } & \text { Main Object } \\
\psi(1+\gamma) & \text { for } & \text { Outlying Background }
\end{array}\right.
$$

In the above equation, "S" denotes the SNR of the primary target return, whereas " $\gamma$ " symbolizes the interference-to-noise ratio (INR) of the outlying target return.

The performance of target detection techniques can be significantly enhanced if the target's radar cross-section (RCS) statistical properties are available. For this reason, Swerling proposed his models (SWJ; J=I-V) to specify the statistical features of the RCS of objects on the basis of $\chi^{2}$-distribution; the degrees of freedom of which are diverse. This model of $2 \kappa$ degrees of freedom roughly treats a target as a large reflector combined with a group of small ones, or a large reflector over a small range of aspect values. It is verified for a wide range of targets and has the feature that it is more strengthened about its mean as $\kappa$ increases.

We start our problem formulation by assuming that Mpulses strike the target under research taking into account that it fluctuates following $\chi^{2}$-model (with $\kappa$ $=1$ ) in its fluctuation. This target will return a statistical signal that can be described by a moment generating function (MGF); the mathematical expression of which takes the form [11]:

$$
\Phi_{y_{0}}(\Omega) \triangleq E_{y_{0}}\left\{\exp \left(-y_{0} \Omega\right)\right\}=\prod_{j=1}^{M} \frac{1}{1+\left(1+\lambda_{j} s\right) \Omega}
$$

In the preceding formula, $y_{0}$ denotes the content of CUT, $E_{x}\{$.$\} stands for the mathematical expectation over$ 
a random variable (RV) $x$, and $\lambda_{i}^{\prime}$ s point to nonnegative eigenvalues of the correlation matrix $\Lambda$.

To statistically formulate this matrix, the target signal is assumed to be of stationary state that allows it to be modeled by first-order Markov chain. Taking this assumption into account, $\boldsymbol{\Lambda}$ tends to take the form of Toeplitz nonnegative definite matrix. Thus, it can be written as [13]:

$$
\Lambda=\left[\begin{array}{ccccccc}
1 & \rho & \rho^{2} & . & . & \rho^{M-2} & \rho^{M-1} \\
\rho & 1 & \rho & . & . & \rho^{M-3} & \rho^{M-2} \\
\rho^{2} & \rho & 1 & . & . & \rho^{M-4} & \rho^{M-3} \\
\cdot & \cdot & \cdot & . & . & . & \cdot \\
\cdot & . & . & . & . & . & . \\
\cdot & \cdot & . & . & . & . & . \\
\rho^{M-2} & \rho^{M-3} & \rho^{M-4} & . & . & 1 & \rho \\
\rho^{M-1} & \rho^{M-2} & \rho^{M-3} & . & . & \rho & 1
\end{array}\right] 0 \leq \rho \leq 1
$$

On the other hand, moderately fluctuating radar targets (i.e. intermediate between SWII and SWI models) represent an important class of fluctuating targets and their detection is of primary concern. This prompts the necessity to investigate the evaluation of $\lambda_{i}$ 's of $\Lambda$ for the partially-correlated case to be verified as Eq.(3) demonstrates. For our analysis to be easily executed, Eq.(1) can be reformatted in a more simpler form as:

$$
\Phi_{y_{0}}(\Omega)=\prod_{\ell=1}^{M} \frac{\vartheta_{\ell}}{\Omega+\vartheta_{\ell}}
$$

According to Eq.(2), the parameter $\vartheta_{\ell}$ is defined as:

$$
\vartheta_{\ell} \triangleq\left\{\begin{array}{lll}
1 & \text { for } & \text { Clear Background } \\
\frac{1}{1+\lambda_{\ell} S}=\zeta_{\ell} & \text { for } & \text { Main Object } \\
\frac{1}{1+\lambda_{\ell} \gamma}=\xi_{\ell} & \text { for } & \text { Outlying Background }
\end{array}\right.
$$

Based on Eq.(5), the PDF of the output of the $\ell^{\text {th }}$ test tap can be obtained by taking its Laplace inverse.

The quality of a radar system is measured with the aid of what is known as figure of merit which has different tools depending on the function being processed. In the world of detection, the major parameters are the probability of detection $\mathrm{P}_{\mathrm{d}}$ and that of false alarm $\mathrm{P}_{\mathrm{fa}}$. In holding other system parameters, enhancing $P_{d}$ is always accompanied with calling for accepting a higher $\mathrm{P}_{\mathrm{fa}}$ as well. Due to its crucial role in attaining a satisfied level of operation, $\mathrm{P}_{\text {fa }}$ possesses the first priority. As a consequence of this, the designer guarantees a certain rate of false alarm and then attempts to ameliorate the level of detection through other means. Actually, $\mathrm{P}_{\mathrm{d}}$ tends to $\mathrm{P}_{\mathrm{fa}}$ if the operating environment is free of any target returns $(S=0)$. Thus, $P_{d}$ is more general than $P_{f a}$. Therefore, we are going to compute it.

Since the noise level estimate $Z$ is statistically varied, average values of $P_{d}$ and $P_{f a}$ are sufficient for the processor performance to be assessed. For $\chi^{2}$-target fluctuation with two-degrees of freedom, the $\mathrm{P}_{\mathrm{d}}$ of partially-correlated case can be calculated as [13]:

$$
\begin{aligned}
& P_{d}=\sum_{j=1}^{M} \prod_{\substack{i=1 \\
i \neq j}}^{M} \frac{\vartheta_{i}}{\vartheta_{i}-\vartheta_{j}} \Theta_{Z}\left(T \vartheta_{j}\right) \\
& \Theta_{Z}(\Omega)=\Phi_{Z}(\Omega) / \Omega
\end{aligned}
$$

Here, $\Theta_{Z}($.$) stands for the Laplace transformation of the$ cumulative distribution function (CDF) of the noise level estimate $Z$. The computation of the constant scale factor $\mathrm{T}$, for a pre-setting rate of false alarm, necessitates the computation of $\mathrm{P}_{\mathrm{fa}}$. As a non-coherent integration of M-pulses is supplied, the needed formula is given by [10]:

$$
P_{f a}=-\left.\frac{1}{\Gamma(M)} \frac{d^{M-1}}{d \Omega^{M-1}}\left\{\frac{\Phi_{Z}(-T \Omega)}{\Omega}\right\}\right|_{\Omega=-1}
$$

$\Gamma(\theta)$ denotes the conventional Gamma function of argument $\theta$. Taking into account that $\Omega=-1$ represents an $\mathrm{M}^{\text {th }}$ order pole of $\Phi_{\mathrm{Z}}(\Omega)$, Eq.(8) can be put in another simpler form as:

$$
P_{f a}=\sum_{j=0}^{M-1} \frac{1}{\Gamma(j+1)} \frac{d^{j}}{d \Omega^{j}}\left\{\Phi_{Z}(-T \Omega)\right\}_{\Omega=-1}
$$

Formulas $(7 \& 9)$ demonstrate that $\Phi_{Z}(\Omega)$ is regarded as the fundamental parameter in evaluating the processor detection performance. Accordingly, the upcoming section is devoted to calculate this crucial parameter for the CFAR variants that are under examination. 


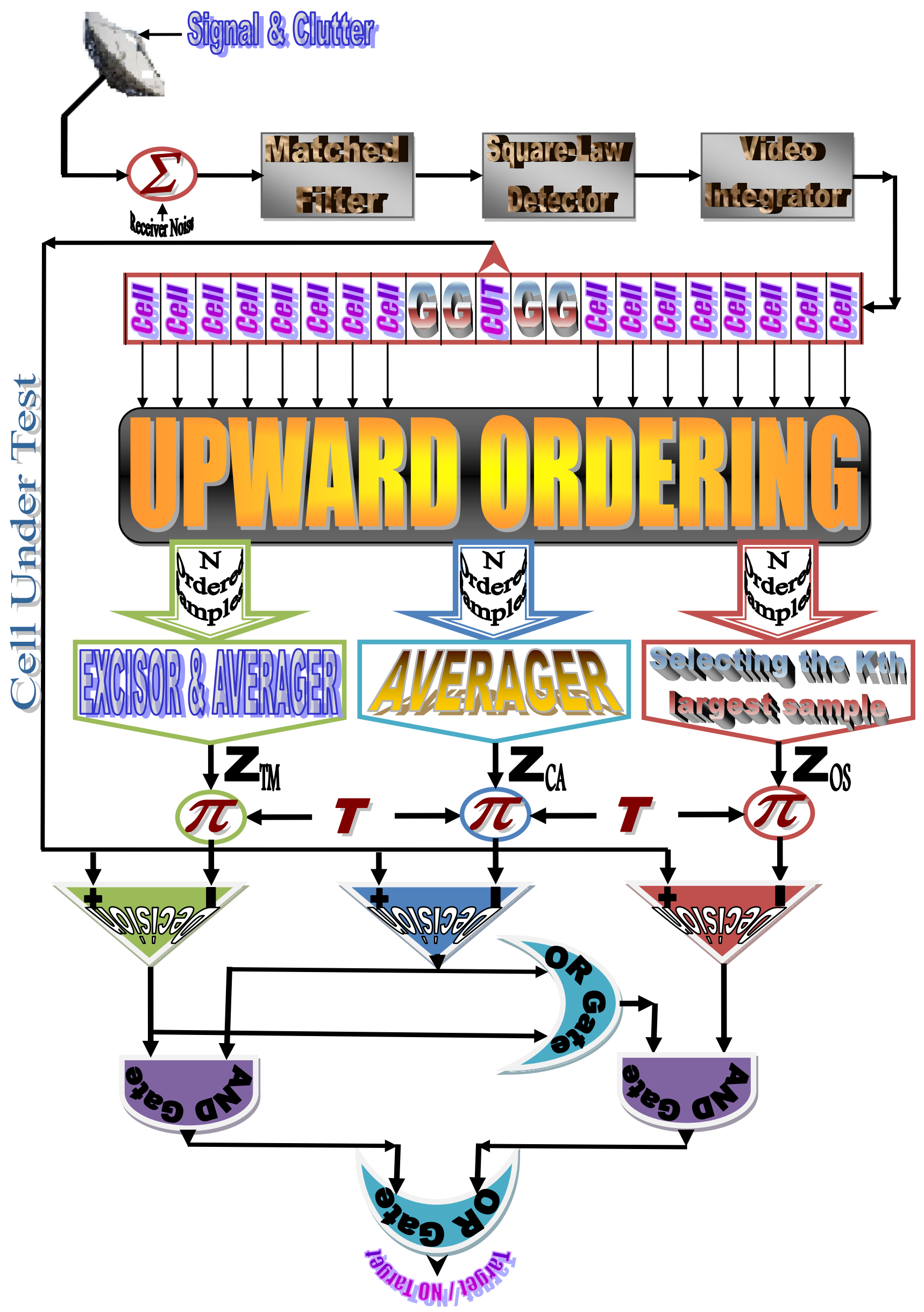

Fig.(1) Archltecture of fusion CA_0S_TI adaptlve approach with postdetection Integration 


\section{Inhomogeneous} Performance Evaluation

Generally, the background clutter is regarded as a significant problem in the performance of radar systems since it introduces more errors in their behavior. To overcome this problem, an attractive class of CFAR alternatives is investigated. In this class, the proper threshold level is selected as a compromise between missing targets and obtaining false alarms, and depends on how important either of these undesired situations is avoided. In addition, this class has been developed with the object of enhancing target detection under different environment circumstances, especially those of regions of clutter edges and those situations of target multiplicity. In the first case, clutter transitions cause problems by generating an excessive number of false alarms, whilst the second scenario is concerned with the case in which one of the targets could be masked when multiple targets exist among the elements of the reference window [14].

The most familiar versions of this class are CA, OS, and TM variants. These models are commonly used as standards against their behavior, the reaction of other developed variants is compared. After evaluating the performance of these algorithms with different test profiles whose samples were Rayleigh distributed, it was found that they exhibit problems when facing the two mentioned situations that may appear in real environments. The hardness of obtaining a solution based on a single CFAR algorithm to cope with diverse noise situations has led to the creation of composite CFAR designing. The analysis of the CA, OS, and TM schemes as well as some of their composite versions is the goal of this section [9].

Normally, the efficiency of the CFAR alternative is measured in the perfect situation of operating conditions or in the presence some of fallacious targets beside the target of interest. Since the ideal case can be considered as a special case of inhomogeneous situation, it is better to analyze the processor performance in heterogeneous background. This is actually the case that we are going to follow in this section.

\subsection{Cell-Averaging (CA)}

The CA detection model is best fit in the environment where the clutter is exponentially distributed and the contents of the reference samples are independent and identically distributed (IID). Under these assumptions, this procedure uses the maximum likelihood estimate of the noise power to set the adaptive threshold. Thus, the CA model is best suited for depicting the behavior of radar system in homogeneous background. Its detection performance is significantly affected when the assumption of homogeneity is violated. In multiple target situations, the noise estimate includes the interfering signal power resulting in an unnecessary increase in overall threshold, which in turn leads to serious degradation in detection probability [6].

In our study of the heterogeneous background for which the elements of the reference set do not obey a common PDF, we are concerned with increases in the value of $\delta$ for some isolated reference cells due to the presence of fallacious targets. In this situation, we assume that some of the reference cells are contaminated with extraneous target returns of the same strength as indicated in Eq.(2), and the remaining cells arise from thermal background. In this situation, the estimated noise level takes the form:

$Z(r, q)=\sum_{i=1}^{r} x_{i}+\sum_{j=1}^{q} y_{j}=X+Y \quad \& \quad r+q=N$

Each one of the RVs $x$ \& $y$ has a MGF of the same form as that given in Eq.(5) taking into account the definition introduced in Eq.(6). Thus, we have:

$$
\Phi_{x}(\Omega)=\prod_{\ell=1}^{M} \frac{\xi_{\ell}}{\Omega+\xi_{\ell}} \& \Phi_{y}(\Omega)=\left(\frac{1}{\Omega+1}\right)^{M}
$$

The RV "X" has $\mathrm{r}$ IID random variables $\mathrm{x}_{\mathrm{i}} \mathrm{s}$, each of them has a MGF of $\Phi_{\mathrm{x}}(\Omega)$ given in Eq.(11). Similarly, $\mathrm{Y}$ is a sum of $\mathrm{q}$ IID random variables $\mathrm{y}_{\mathrm{j}} \mathrm{s}, \Phi_{\mathrm{y}}(\Omega)$ denotes the MGF of each one as Eq.(11) indicates. Therefore, the MGF of $Z$ can be easily obtained as:

$$
\Phi_{C A}(\Omega)=\left(\frac{1}{\Omega+1}\right)^{M q}\left(\prod_{\ell=1}^{M} \frac{\xi_{\ell}}{\Omega+\xi_{\ell}}\right)^{r}
$$

As soon as the MGF of the CA processor is calculated, the false alarm and the detection probabilities are easily evaluated as Eqs.(7 \& 9) reveal.

\subsection{Order-Statistics (OS)}

The OS scheme is an amended form of CA. It is primarily designed for a situation in which several targets, in a particular area, simultaneously reflect signals, of similar or different amplitudes, at the entrance of the detector. It has no need for guard cells and can even include the CUT itself among the candidates of the reference window without making self target cancellation. Also, the threshold is unaffected by targets and the targets do not mask each other. This variant of CFAR mechanisms is robust and well performing in both inhomogeneous clutter and multi-target situations. The major drawback of OS scenario is the high processing power required to perform the sorting technique [7].

This category of CFAR algorithms estimates the noise level by extracting the $\mathrm{K}^{\text {th }}$ largest sample among the elements of the reference window. To carry out this extraction, the reference cells are firstly sorting in an 
ascending order, in an accordance with their magnitudes, in such a way that:

$$
\mathrm{Y}_{(\ell)} \leq \mathrm{Y}_{(\ell+1)} \quad \& \quad \ell \in[1,2, \ldots \ldots, \mathrm{N}-1]
$$

In this ordered samples, $Y_{(1)}$ is regarded as the lowest noise level whereas $Y_{(\mathrm{N})}$ represents the highest level. After the ranking process, it is convenient to excise one or more of the highest samples and pick the $\mathrm{K}^{\text {th }}$ ordered sample to constitute the unknown noise power level. Thus, its test statistic takes the form:
$Z_{O S}=Y_{(K)} \quad \& \quad K \in[1,2, \ldots \ldots, N]$

For the OS performance to be evaluated, the PDF of the $\mathrm{K}^{\text {th }}$ order-statistics out of $\mathrm{N}$ samples is needed, given that the samples are independent but not identically distributed. In the case where the reference window contains several extraneous target returns, let's assume that there are $\mathrm{r}$ outlying target returns with power level of $\psi(1+\gamma)$ and $\mathrm{q}=\mathbf{N}-\mathrm{r}$ cells from clear background with noise level of $\psi$. As a consequence of this assumption, the CDF of the $\mathrm{K}^{\text {th }}$ ordered sample is given by [15]:

$F_{K}^{N H}(t ; N, r)=\sum_{i=K}^{N} \sum_{j=\operatorname{Max}(0, i-r)}^{\operatorname{Min}(i, q)}\left(\begin{array}{c}q \\ j\end{array}\right)\left(\begin{array}{c}r \\ i-j\end{array}\right) \sum_{n=0}^{j}\left(\begin{array}{l}j \\ n\end{array}\right)(-1)^{n} \sum_{m=0}^{i-j}\left(\begin{array}{c}i-j \\ m\end{array}\right)(-1)^{m}\left\{1-F_{C}(t)\right\}^{q-n}\left\{1-F_{O}(t)\right\}^{R-m}$

In the above formula, $F_{C}$ (.) symbolizes the CDF of the cell that contains clear background whereas $F_{O}($.) denotes the same thing for the cell that has outlying target return. The RV's representing returns from clutter background and extraneous targets have $\Phi_{\mathrm{y}}(\Omega)$ $\& \Phi_{\mathrm{x}}(\Omega)$, respectively, for their MGFs as that given in Eq.(11). As Eq.(7) demonstrates, the Laplace transformation of the CDF of $\mathrm{y}$ is given by its MGF divided by $\Omega$. Thus, $F_{C}($.) has a mathematical form given by:

$$
F_{C}(t)=1-\sum_{j=0}^{M-1} \frac{t^{j}}{\Gamma(j+1)} e^{-t} U(t)
$$

$$
F_{o}(t)=L^{-1}\left\{\frac{1}{\Omega} \prod_{\ell=1}^{M} \frac{\xi_{\ell}}{\Omega+\xi_{\ell}}\right\}
$$

In the preceding expression, $\mathrm{L}^{-1}$ stands for the Laplace inverse operator. The Laplace inverse processing yields:

$$
\begin{gathered}
F_{o}(t)=1-\sum_{j=1}^{M} \theta_{j} \exp \left(-\xi_{j} t\right) U(t) \\
\theta_{j} \triangleq \prod_{\substack{i=1 \\
i \neq j}}^{M} \frac{\xi_{i}}{\xi_{i}-\xi_{j}}
\end{gathered}
$$

The substitution of Eqs.(16 \& 18) into Eq.(15) results in:

On the same manner, $F_{O}($.$) can be calculated as:$

$$
\left.\begin{array}{rl}
F_{K}^{N H}(t ; N, r)= & \sum_{i=K}^{N} \sum_{j=\max (0, i-r)}^{\min (i, q)}\left(\begin{array}{l}
q \\
j
\end{array}\right)\left(\begin{array}{c}
r \\
i-j
\end{array}\right) \sum_{\varepsilon=0}^{j} \sum_{\ell=0}^{i-j}\left(\begin{array}{c}
j \\
\varepsilon
\end{array}\right)\left(\begin{array}{c}
i-j \\
\ell
\end{array}\right)(-1)^{i-\varepsilon-\ell}\left\{\sum_{m=0}^{M-1} \frac{t^{m}}{\Gamma(m+1)} e^{-t}\right\}^{q-\varepsilon} \\
\left\{\sum_{n=1}^{M} \theta_{n} e^{-\xi_{n} t}\right.
\end{array}\right\}
$$

With the aid of binomial theorem, the bracketed quantities can be expanded as a polynomial of $t$.

Following this procedure of expansion, Eq.(19) can be rewritten as:

$$
\begin{aligned}
& F_{K}^{N H}(t ; N, r)=\sum_{i=K}^{L} \sum_{j=\max (0, i-r)}^{\min (i, q)}\left(\begin{array}{c}
q \\
j
\end{array}\right)\left(\begin{array}{c}
r \\
i-j
\end{array}\right) \sum_{n=0}^{j}\left(\begin{array}{l}
j \\
n
\end{array}\right) \sum_{m=0}^{i-j}\left(\begin{array}{c}
i-j \\
m
\end{array}\right)(-1)^{i-n-m} \sum_{u_{0}=0}^{q-n} \sum_{u_{1}=0}^{q-n} \ldots \ldots \ldots \ldots \sum_{u_{M-1}=0}^{q-n} \\
& \frac{\Xi\left(q-n ; u_{0}, u_{1}, u_{2}, \ldots \ldots \ldots, u_{M-1}\right)}{\prod_{\sigma=0}^{M-1}[\Gamma(\sigma+1)]^{u_{\sigma}}} \sum_{v_{1}=0}^{r-m} \sum_{v_{2}=0}^{r-m} \ldots \ldots \ldots . . . \sum_{v_{M}=0}^{r-m} \Xi\left(r-m ; v_{1}, v_{2}, \ldots \ldots, v_{M}\right) \prod_{\eta=1}^{M}\left(\theta_{\eta}\right)^{v_{\eta}} \\
& t^{\sum_{\tau=0}^{M-1} \tau u_{\tau}} \exp \left(-\left(q-n+\sum_{\delta=1}^{M} v_{\delta} \xi_{\delta}\right) t\right)
\end{aligned}
$$

Finally, the Laplace transformation of Eq.(20) leads to: 


$$
\begin{aligned}
\Theta_{K}^{N H}(\Omega ; N, r) & =\sum_{i=K}^{N} \sum_{j=\max (0, i-r)}^{\min (i, q)}\left(\begin{array}{l}
q \\
j
\end{array}\right)\left(\begin{array}{c}
r \\
i-j
\end{array}\right) \sum_{n=0}^{j}\left(\begin{array}{l}
j \\
n
\end{array}\right) \sum_{m=0}^{i-j}\left(\begin{array}{c}
i-j \\
m
\end{array}\right)(-1)^{i-n-m} \sum_{u_{0}=0}^{q-n} \sum_{u_{1}=0}^{q-n} \ldots \ldots \ldots \ldots \sum_{u_{M-1}=0}^{q-n} \\
& \frac{\Xi\left(q-n ; u_{0}, u_{1}, u_{2}, \ldots \ldots ., u_{M-1}\right)}{\prod_{\sigma=0}^{M-1}[\Gamma(\sigma+1)]^{u_{\sigma}}} \sum_{v_{1}=0}^{r-m} \sum_{v_{2}=0}^{r-m} \ldots \ldots \ldots \sum_{v_{M}=0}^{r-m} \Xi\left(r-m ; v_{1}, v_{2}, \ldots \ldots, v_{M}\right) \prod_{\eta=1}^{M}\left(\theta_{\eta}\right)^{v_{\eta}} \\
& \frac{\Gamma\left(\sum_{\tau=0}^{M-1} \tau u_{\tau}+1\right)}{\left(\Omega+q-n+\sum_{\delta=1}^{M} v_{\delta} \xi_{\delta}\right)^{\left(\sum_{\tau=0}^{M-1} \tau u_{\tau}+1\right)}}
\end{aligned}
$$

In the previous formulas, the factor $\Xi\left(\mathrm{Q} ; \mathrm{q}_{1}, \mathrm{q}_{2}, \ldots \ldots, \mathrm{q}_{\mathrm{M}}\right)$ has a form given by:

$$
\Xi\left(Q ; q_{1}, \ldots, q_{M}\right) \triangleq\left\{\begin{array}{lll}
\frac{\Gamma(Q+1)}{\prod_{i=1}^{M} \Gamma\left(q_{i}+1\right)} & \text { if } & \sum_{j=1}^{M} q_{j}=Q \\
0 & \text { if } & \sum_{j=1}^{M} q_{j} \neq Q
\end{array}\right.
$$

As a function of $W_{i}{ }^{\prime} s$, Eq.(23) can be rewritten as:

$Z_{T M}\left(T_{L}, T_{U}\right)=\sum_{j=1}^{N_{T}}\left(N_{T}-j+1\right) W_{j} \& N_{T} \triangleq N-T_{L}-T_{U}$

In terms of the $\Omega$-domain representation of the CDF of $\mathrm{Y}_{(\mathrm{i})}$ 's, the MGF of the random variables $W_{j}$ 's becomes [10]:

$$
\Phi_{W_{j}}(\Omega)=\left\{\begin{array}{l}
\Omega \Theta_{T_{L}+1}^{N H}(\Omega ; N, r) \text { for } \ell=1 \\
\frac{\Theta_{T_{L}+\ell}^{N H}(\Omega ; N, r)}{\Theta_{T_{L}+\ell-1}^{N H}(\Omega ; N, r)} \text { for } 1<\ell \leq N_{T}
\end{array}\right.
$$

Since the new random variables $W_{j}$ 's are statistically independent, the MGF of the noise level estimate $Z_{T M}$ is simply the product of the MGF of each one of its elements. Thus,

$\Phi_{Z_{T M}}\left(\Omega ; T_{L}, T_{U}\right)=\left.\prod_{\ell=1}^{N_{T}} \Phi_{W_{\ell}}(\Omega)\right|_{\Omega=\left(N_{T}-\ell+1\right) \Omega}$

The major disadvantage of TM scheme is, as previously mentioned in OS, the high processing power required for performing the sorting algorithm. To mitigate this problem, one of the possible solutions is based on splitting the reference set into two symmetrically subsets about the CUT. The cells of each subset are processed and the statistic $Z$ may be chosen by further processing the two subsets outputs. The subset output is simply the trimmed sum of its ordered range cells. The final estimate of the noise power is obtained by taking the mean value of the two noise

Clearly, the order statistics $Y_{(I)},,, \ldots, Y_{(N)}$ are not IID random variables even if the original samples $\mathbf{y}_{1}, \ldots$, $\mathrm{y}_{\mathrm{N}}$ are IID. However, on the assumption that the samples $\mathrm{y}_{1}, \mathrm{y}_{2}, \ldots, \mathrm{y}_{\mathrm{N}}$ are IID and exponentially 
level estimates of the two subsets. Actually, this idea may lead to reduction in overall computation time compared to one set TM variant, because the ranking is performed in subsets of smaller sizes. In this situation, assume that $r_{1}$ cells contain outlying target returns, $\mathrm{N} / 2-\mathrm{r}_{1}$ ones immersed in thermal noise, $\alpha_{\mathrm{L}}$ censored cells from the lower end and $\alpha_{U}$ excised samples from the upper end of its order statistics. Similarly, the lagging is assumed to have $r_{2}$ interfering cells, N/2- $r_{2}$ clear samples, its associated order statistics is trimmed from its ends, where the lowest $\beta_{\mathrm{L}}$ ordered cells are removed and $\beta_{U}$ largest ranked cells are nullified. Under these circumstances, the MGF's of their noise power level estimates, $Z_{1}$ and $Z_{2}$, have the same form as that given by Eq.(27) after replacing its argument with the corresponding parameter values for the leading and lagging subsets. Finally, the two noise level estimates are combined through the mean-level operation to investigate the final noise level estimate. Thus,

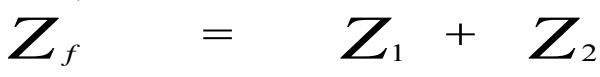

Since the two noise level estimates are statistically independent, the $Z_{f}$ has a MGF given by:

$$
\Phi_{Z_{f}}(\Omega)=\Phi_{Z_{T M}}\left(\Omega ; \alpha_{L}, \alpha_{U}\right) \Phi_{Z_{T M}}\left(\Omega ; \beta_{L}, \beta_{U}\right)
$$

As soon as the $\Omega$-domain representation of the PDF of the resultant noise level estimate is investigated, the processor detection performance is completely evaluated, as Eq.(7) manifests.

\subsection{CA_OS \& CA_TM-CFAR Developed Versions}

A considerable amount of work on single sensor CFAR signal detection has been done. Owing to the diversity of the radar search environment (target multiplicity and clutter edges), there exists no universal CFAR strategy. The ruggedness of finding an efficient CFAR algorithm is the prime motivation for developing a new structure of adaptive techniques. In this regard, the use of multiple sensors is widely increasing in surveillance systems. The main goal of employing this technology is to enhance the system reliability and speed of reaction, along with achieving a larger area of coverage. In this technology, the developed CFAR processors is designed to exploit the merits of the distinguished algorithms: one has good homogeneous behavior whilst the other gives satisfied inhomogeneous reaction in order to benefit the high performance in both cases of the operating conditions along with maintaining the desired rate of false alarm. This category can be implemented by parallel operation of the selected types of CFAR variants. Thus, the CA model is combined with either OS or TM scheme to emerge CA_OS or CA_TM new modes in the world of adaptive detection. In any one of them, the two candidates carry out their operations simultaneously and independently in such a way that the adjustment factor $\mathrm{T}$ is common for achieving their own detection threshold against which the content of CUT is compared to independently decide the presence/absence of the primary target. The final decision is taken in fusion center which is represented by an "AND" gate circuit. In accordance with the truth table of the AND gate, the presence of the target in the CUT is decided iff both of the input single decisions of the fusion center are positive. Otherwise, the fusion center's decision is negative and target is not at the location which corresponds to the CUT [13]. In other words, the final decision is attained according to:

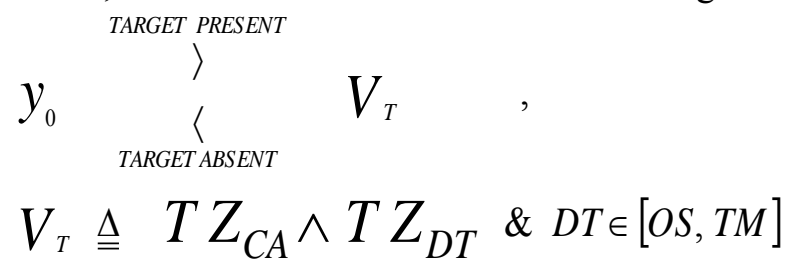

In the preceding formula, " $y_{0}$ " denotes the content of the CUT, " $V_{T}$ " stands for the detection threshold, $Z_{\mathrm{DT}}$ designates the background estimate by the detector DT, and $" \wedge "$ symbolizes the algebraic Boolean of AND gate. Since the two single decisions are statistically independent, the global false alarm and detection probabilities can be mathematically formulated as:

$P_{f a}^{C A D T}=P_{f a}^{C A} P_{f a}^{D T}, P_{d}^{C A D T}=P_{d}^{C A} P_{d}^{D T} \& D T \in[O S, T M](31)$

As a function of the performance of CA, OS, and TM procedures, the performances of CA_OS and CA_TM novel models are completely evaluated, as Eq.(31) indicates.

\subsection{Fusion CA_OS_TM-CFAR Emerged Strategy}

A robust detector should not only pick out targets but also diminish false alarms. For target detection in complex situations, it's hard to attain high detection performance simultaneously with maintaining low rate of false alarm. An effective target detector requires a combination of different features in such a way that each feature resolves one of the challenges that enface the detection characteristics. In this regard, more reliable detection results can be realized by fusion processing. In other words, an architecture involving decentralized processing at multiple sensor locations provides the proper choice for optimum results in heterogeneous cases of operation. Such construction involves higher 
reliability and survivability, improved system performance at low latency. In this category of fusion technology, a new pattern of CA, OS, and TM processors has been recently emerged [.]. Each one of these local detectors carries out its local binary decision, and sends it to the fusion center which achieves the overall decision based on the local decisions. This overall decision is acquired depending on the 'AND' and 'OR' fusion rules [17].

As its designation indicates, the developed fusion approach relies on individual operation of CA, OS, and TM scenarios. Fig.(1) portrays the detailed architecture of the developed model. In this scene, there are three individual arms; each one is associated with one of the standard detectors. Depending on the required rate of false alarm, the clutter level estimate along with the signal strength in the CUT of each detection scheme is used to build a decision about the presence/absence of the target in the CUT. According to the appropriate fusion rule, the three local decisions are simultaneously mixed in the fusion center to arrive at the final decision. As the circuit in Fig.(1) shows, the potential outputs of fusion CA_OS_TM strategy are summarized in Table I. Owing to the providing of the CA scheme of a low false alarm rate and a high detection probability, its output is taken as a reference for the fusion center. When the CA output is 1 (presence of target), there is a possibility of occurring false alarm, caused by clutter transition or multi-targets. To eliminate this eventuality, the AND fusion rule 1, indicated in Eq.(32), can be applied. This rule necessitates the application of an AND logic between the CA output and that obtained by applying an OR logic between the outputs of the OS and TM scenarios. On the other hand, when the CA output is 0 (absence of target), there exists the possibility of a target lost caused by clutter

$$
\begin{aligned}
P_{D G} & =\left(1-P_{d_{C A}}\right) P_{d_{O S}} P_{d_{T M}}+P_{d_{C A}}\left(1-P_{d_{O S}}\right) P_{d_{T M}}+P_{d_{C A}} P_{d_{O S}}\left(1-P_{d_{T M}}\right)+P_{d_{C A}} P_{d_{O S}} P_{d_{T M}} \\
= & P_{d_{C A}}\left(P_{d_{O S}}-2 P_{d_{O S}} P_{d_{T M}}+P_{d_{T M}}\right)+P_{d_{O S}} P_{d_{T M}}
\end{aligned}
$$

All the parameters of the above formula are previously handled. So, the detection performance of the fusion CA_OS_TM is completely analyzed. Our scope in the upcoming section is to numerically simulate the derived formulas through a PC device using $\mathrm{C}++$ programming language to obtain an idea about the new contribution of the novel version of adaptive strategies.

\section{Processor Performance Assessment}

Here, our object is mainly interested in numerically substantiating the effectiveness of our analysis for the CFAR algorithms under examination through the presentation of some numerical plots. In addition, it is interference. To overcome this problem, we can apply the AND fusion rule 2, exhibited in Eq.(32). This necessitates the application of an AND logic between the outputs of OS and TM variants.

Rule1 $=\mathrm{CA} \wedge(\mathrm{OS} \vee \mathrm{TM}) \&$ Rule $2=\mathrm{OS} \wedge \mathrm{TM}(32)$

In the preceding formula, " $\wedge$ " stands for the algebraic Boolean of AND gate whilst " $\vee$ " symbolizes the same thing of OR gate.

Table 1 Possible Outputs of Fusion CA_OS_TM

\begin{tabular}{|c|c|c|c|}
\hline $\begin{array}{c}\text { CA } \\
\text { Scenario }\end{array}$ & $\begin{array}{c}\text { OS } \\
\text { Procedure }\end{array}$ & $\begin{array}{c}\text { TM } \\
\text { Strategy }\end{array}$ & $\begin{array}{c}\text { FUSION } \\
\text { RULE }\end{array}$ \\
\hline 0 & 0 & 0 & 0 \\
\hline 0 & 0 & 1 & 0 \\
\hline 0 & 1 & 0 & 0 \\
\hline 0 & 1 & 1 & 1 \\
\hline 1 & 0 & 0 & 0 \\
\hline 1 & 0 & 1 & 1 \\
\hline 1 & 1 & 0 & 1 \\
\hline 1 & 1 & 1 & 1 \\
\hline
\end{tabular}

The outcomes of rows $4,6,7 \& 8$, refer to the events that corresponding to the presence of the target of interest. Since the occurrence of one of them excludes the occurrence of the others, they are mutually exclusive. Taking in mind that the decisions of CA, OS, and TM procedures are also independent events, the global detection probability " $\mathrm{P}_{\mathrm{DG}}$ " of the new implementation can be evaluated, in accordance with the Boolean algebra, as:

of importance to take an idea about the behavior of the new methodology of combination of the standard CFAR scenarios and to what extent this new category of adaptive schemes can enhance the performance of their original versions if the operating conditions are held the same. To address this objective, it is of primary concern to examine the performance of the novel strategy, as well as its conventional procedures, in the case where the operating environment is free of, or contaminated with fallacious target returns when these targets along with the target of interest fluctuate and following $\chi^{2}$-distribution with two-degrees of freedom in their fluctuation. Unless otherwise specified, the reference window is assumed to have $24(\mathrm{~N}=24)$ cells, 
and the tested CFAR variants are designed to guarantee a rate of false alarm of $10^{-6}$. For the obtained results to be comprehensive, we will designate the fusion CA_OS_TM algorithm as linear fusion (LF) in all the plots displayed in this research. Additionally, for the results to be easily understandable, we will go to categorize them into groups; the elements of each group have a common characteristic that differentiates its curves from those of the others. Since the estimation of the final background level is achieved through the mean-level operation and this is common for all the processors listed here, it is not necessary to indicate this property in the processor representation. Therefore, the abbreviation refers to the mean-level (ML) is eliminated from all the tested procedures. In other words, in the forthcoming scenes, each alternative will be nominated by the rule based on which the noise level is estimated from each sub-window. In this regard, a designation like $\operatorname{TM}\left(\mathrm{N}_{1}, \mathrm{~N}_{2}\right)$ on a particular curve means that it is associated with a CFAR procedure the threshold of which is constructed by weighting the ML of symmetrically adding the candidates of each reference subset after rejecting $\mathrm{N}_{1}$ lower ordered cells and $\mathrm{N}_{2}$ top sorted ones from its samples. Throughout our presentation, all the numerical results are achieved for the optimum parameter values, where $\left(\mathrm{N}_{1}=\mathrm{N}_{2}=2\right)$ in the case of TM scheme, $\left(K_{1}=K_{2}=10\right)$ in the case of OS algorithm, and $\left(r_{1}=r_{2}=1, \rho_{\mathrm{p}}=\rho_{\mathrm{s}} \& \mathrm{INR}=\mathrm{SNR}\right)$ in multitarget situations where $\rho_{p}$ represents the correlation coefficient among the primary target returns whilst $\rho_{\mathrm{s}}$ denotes the same thing for the secondary target returns. Let's now go to briefly discuss the behavior of the curves of each plot among the candidates of every category.

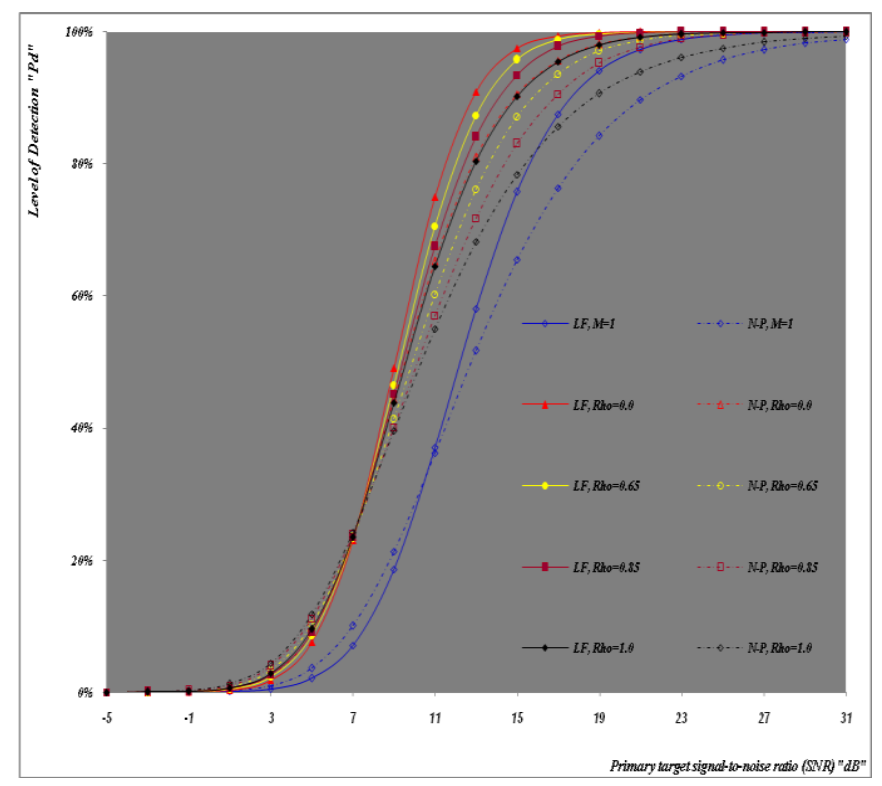

Fig.2 Multi-pulse homogeneous detection performance of
LF-CFAR scheme for $\chi^{2}$-fluctuating targets with twodegrees of freedom when $\mathrm{N}=\mathbf{2 4}, \mathrm{M}=2$, and $\mathrm{P}_{\mathrm{fa}}=10^{-6}$

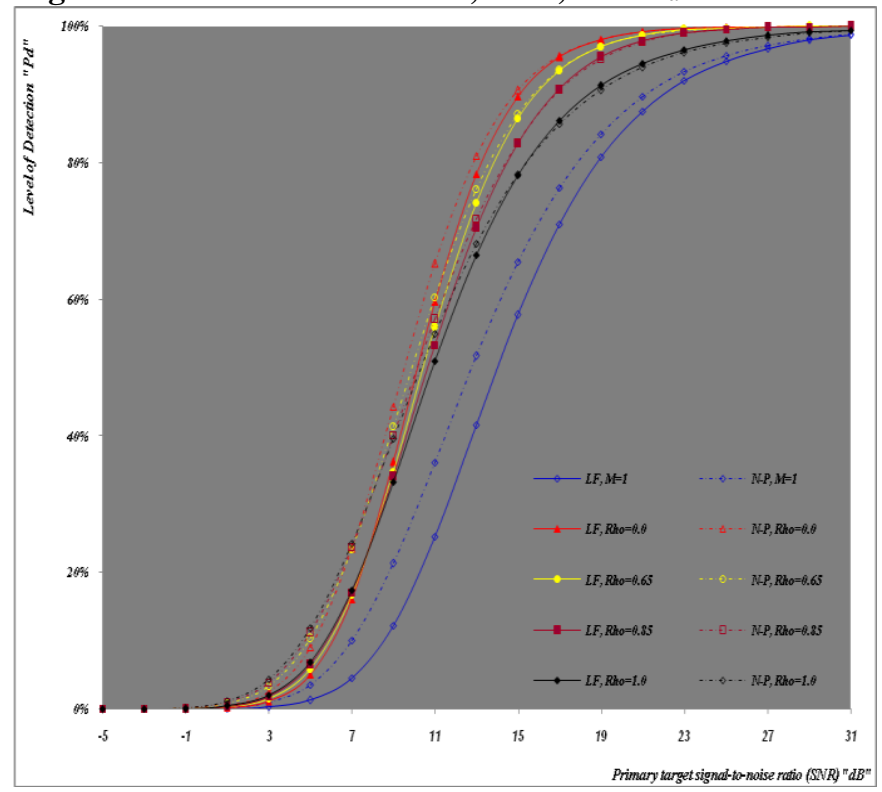

Fig.3 Multi-pulse multi-target detection performance of LF-CFAR scheme for $\chi^{2}$ fluctuating targets with twodegrees of freedom when $N=24, M=2, r_{1}=r_{2}=1, I N R=S N R$, and $\mathrm{P}_{\mathrm{fa}}=\mathbf{1 0}^{-6}$

The first category is devoted to the more interesting characteristics that measure the ability of the adaptive scheme to satisfactory decide the presencelabsence of the interesting target in an environment which has homogeneous or inhomogeneous background. This is known as detection characteristic in the radar terminology. This class of shows is depicted by graphing the level of detection $\left(\mathrm{P}_{\mathrm{d}}\right)$ as a function of the strength of the primary target return (SNR), for a specified parameter values, as Figs.(2-9) illustrate. The performance of the Neymann-Pearson (N-P) detector, as a reference of comparison, is attached to the contents of each one of these figures. Additionally, to see to what extent the non-coherent integration can improve the processor performance, the single-sweep $(\mathrm{M}=1)$ characteristics of both N-P and the examined variant are incorporated with the curves of each one of these plots. This category of figures consists of eight plots with two scenes, one is associated with homogeneous while the other is concerned with inhomogeneous situations, for each processor (LF, CA, $\mathrm{OS}(10), \operatorname{TM}(2,2))$. The candidates of these figures are labeled with the underlined processor along with the intensity of correlation $(\rho)$ or the number of integrated pulses $(M)$. Figs. $(2 \& 3)$ are devoted to the LF methodology to see its contribution in detecting partially-correlated $\chi^{2}$ targets in the absence, Fig.(2), and in the presence of spurious targets, Fig.(3). For weak signal strength (SNR), it is noted that the processor performance enhances as the correlation strength increases and this behavior is rapidly altered as the target return becomes 
strengthened where the detection probability increases as the coefficient of correlation among target returns tends to be diminished. Meanwhile, the N-P performance is higher than that of the LF for weak target return and this reaction is quickly reversed as the strength of the signal return tends to be stronger, where the LF performance outweighs that of N-P for higher SNR values. This feedback is observed either noncoherent integration is absent $(\mathrm{M}=1)$ or it takes place $(\mathrm{M}>1)$; given that the LF operates in an ideal (homogeneous) environment, as Fig.(2) portrays. Additionally, the partially-correlated cases are embrassed by Swerling models, SWII $(\rho=0)$ \& SWI $(\rho=1.0)$, and this behavior is common either the processor is optimum (N-P) or adaptive (LF). Moreover, the point of alteration is shifted towards lower SNR values as $\mathrm{M}$ augments. Furthermore, the point of revision of LF methodology coincides with that of N-P detector. In multi-target situations, on the other hand, the reaction of LF strategy is always inferior than that of N-P procedure in the absence of non-coherent integration $(\mathrm{M}=1)$ and the two performances tend to be the same as signal strength becomes more stronger $(\mathrm{SNR}=30 \mathrm{~dB})$ as Fig.(3) delineates. In the situation of non-coherent integration, the curves of this plot behave like, in their variation, the corresponding ones in Fig.(2) with the exception that the point of commutation of LF scenario is different from that of the N-P scheme. In addition, the detection performance of N-P is always superior than that of LF variant for weak and modest signal strength. However, the LF multi-target reaction tends to be coincident with that of N-P and may surpass it as the primary target returns become stronger (SNR $\geq$ $17 \mathrm{~dB}$ ), as Fig.(3) depicts. The results portrayed in Figs.(2 \& 3) confirm the utility of using non-coherent integration in improving the performance of an adaptive processing mechanism, either the operating environment is homogeneous or contaminated with outlying target returns.

After discussing the performance of the LF strategy in facing different operating conditions, let us now turn our attention to its fundamental versions of CFAR processing. Figs. $(4 \& 5)$ are associated with the standard CA model. Fig.(4) depicts the CA performance in homogeneous background whilst Fig.(5) exhibits the same thing in the presence of interfering target returns along with those of the target of interest. The candidates of these plots have the same behavior as their corresponding ones in Fig.(2\& 3) with some modifications. Firstly, the performance of N-P is always higher than that of $\mathrm{CA}$ either $\mathrm{M}=1$ or $\mathrm{M}=2$ as well as either the background is homogeneous or inhomogeneous. For $\mathrm{M}>1$, the points of performance alteration are located at approximately the same signal strength with higher detection level in the case of N-P processor. However, in multi-target situation, the two points are different with higher signal strength as well as higher detection level in the case of the CA variant. The target multiplicity performance of CA is very weak with higher gain via the introduction of non-coherent integration.

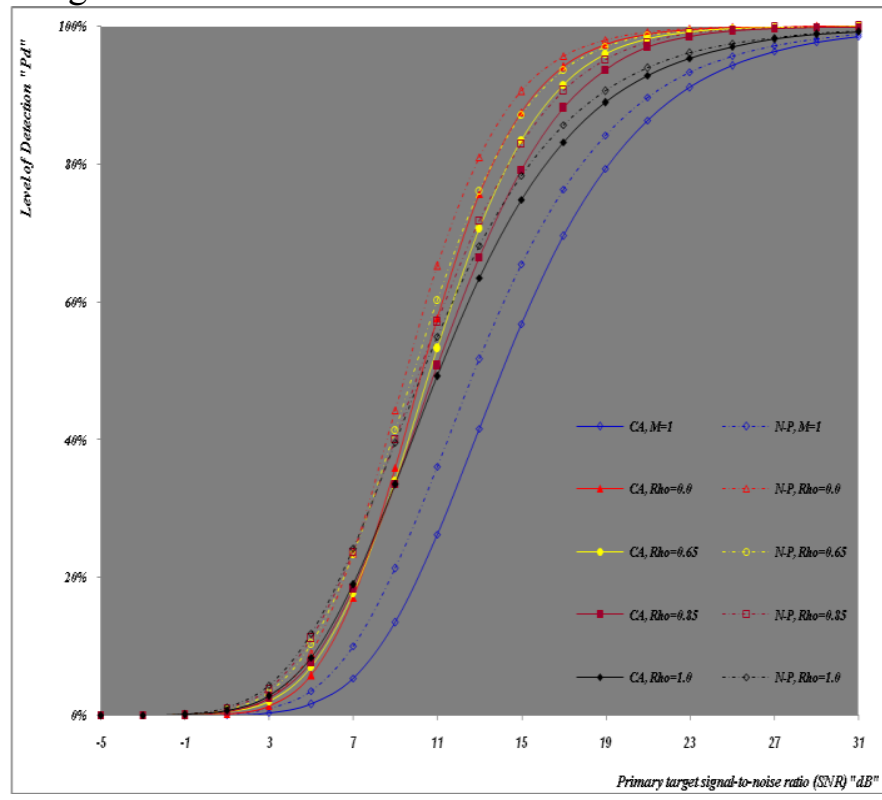

Fig.4 Multi-pulse homogeneous detection performance of CA-CFAR scheme for $\chi^{2}$-fluctuating targets with twodegrees of freedom when $N=24, M=2$, and $P_{f_{a}}=10^{-6}$

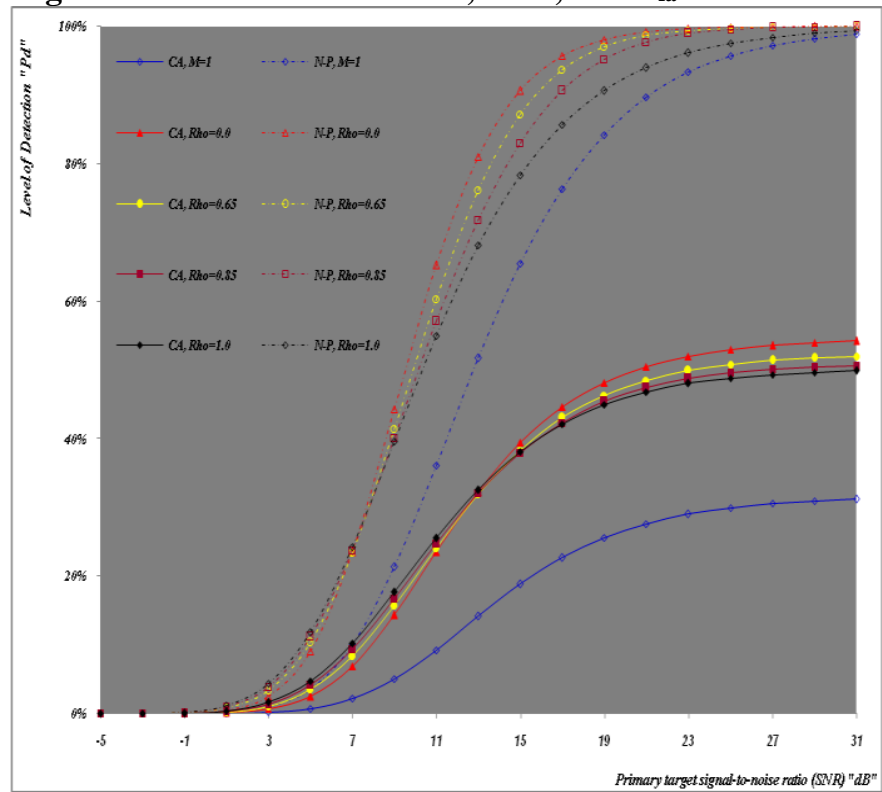

Fig.5 Multi-pulse multi-target detection performance of CA-CFAR scheme for $\chi^{2}$ fluctuating targets with twodegrees of freedom when $N=24, M=2, r_{1}=r_{2}=1, I N R=S N R$, and $P_{\mathrm{fa}_{\mathrm{a}}}=\mathbf{1 0}^{-6}$

The second standard scheme in constructing LF strategy is the OS procedure. The homogeneous and inhomogeneous detection behaviors are illustrated in Figs.(6 \& 7). In homogeneous situation, the same noted remarks about the reaction of CA detector can be clearly observed in the curve variations of Fig.(6) with minor degradation, i.e. with lower detection probability 
of the OS scheme relative to that of the CA variant. In multi-target background, on the other hand, the OS processor exhibits a detection performance which is comparable to (relative to that of the CA mode) that of the N-P scheme with large gap (from the SNR point of view) in mono-pulse $(\mathrm{M}=1)$ case and this gap is reduced as $M$ increases.

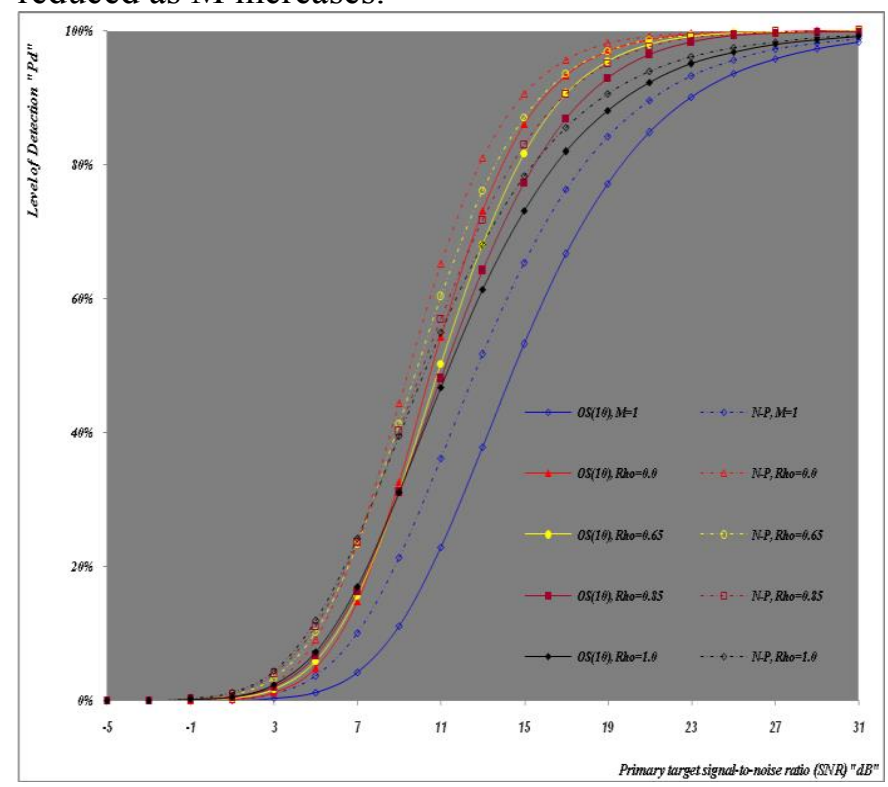

Fig.6 Multi-pulse homogeneous detection performance of OS(10)-CFAR scheme for $\boldsymbol{\chi}^{2}$-fluctuating targets with twodegrees of freedom when $\mathrm{N}=24, \mathrm{M}=2$, and $\mathrm{P}_{\mathrm{fa}}=10^{-6}$

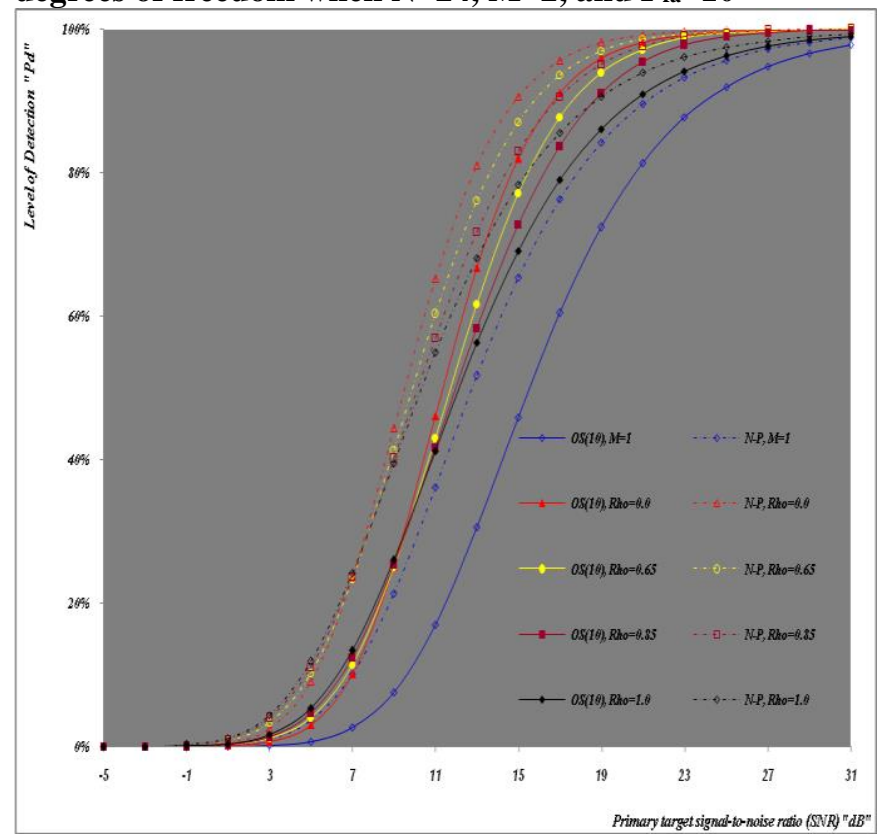

Fig.7 Multi-pulse multi-target detection performance of OS(10)-CFAR scheme for $\chi^{2}$ fluctuating targets with two-degrees of freedom when $\mathrm{N}=24, \mathrm{M}=2, \mathrm{r}_{1}=\mathrm{r}_{2}=1$, $\mathrm{INR}=\mathrm{SNR}$, and $\mathbf{P}_{\mathrm{fa}}=\mathbf{1 0}^{-6}$

The last standard version is the TM technique. Figs.(8 \& 9) are devoted to its performance evaluation. Fig.(8) discusses the homogeneous performance whilst Fig.(9) portrays the inhomogeneous behavior under the same stated conditions.

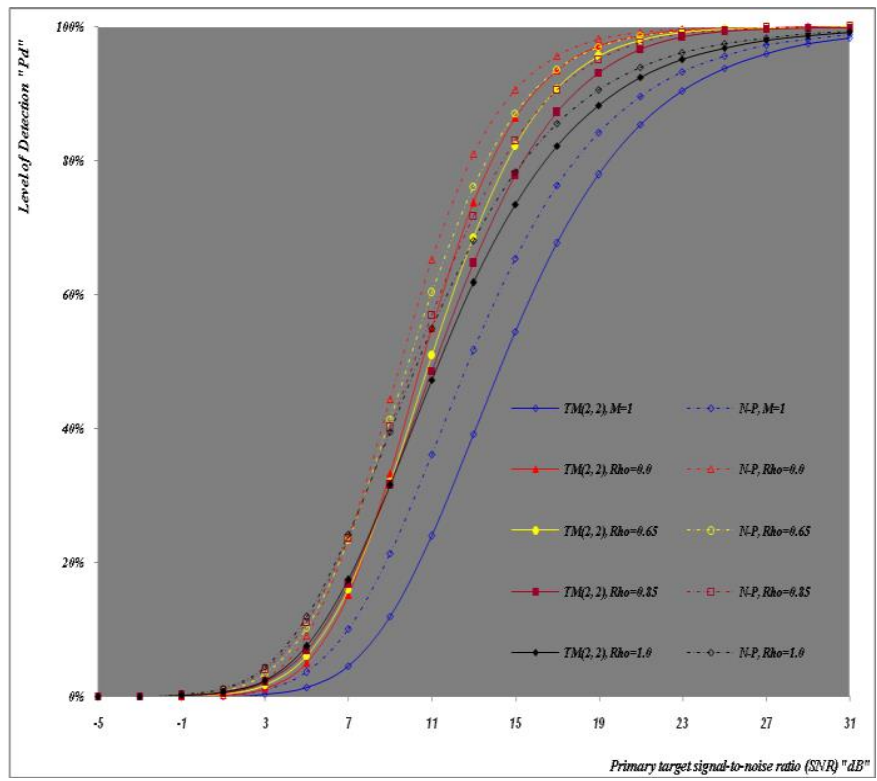

Fig.8 Multi-pulse homogeneous detection performance of $\operatorname{TM}(2,2)-C F A R$ scheme for $\chi^{2}$-fluctuating targets with two-degrees of freedom when $\mathrm{N}=24, \mathrm{M}=2$, and $\mathrm{P}_{\mathrm{fa}}=10^{-6}$

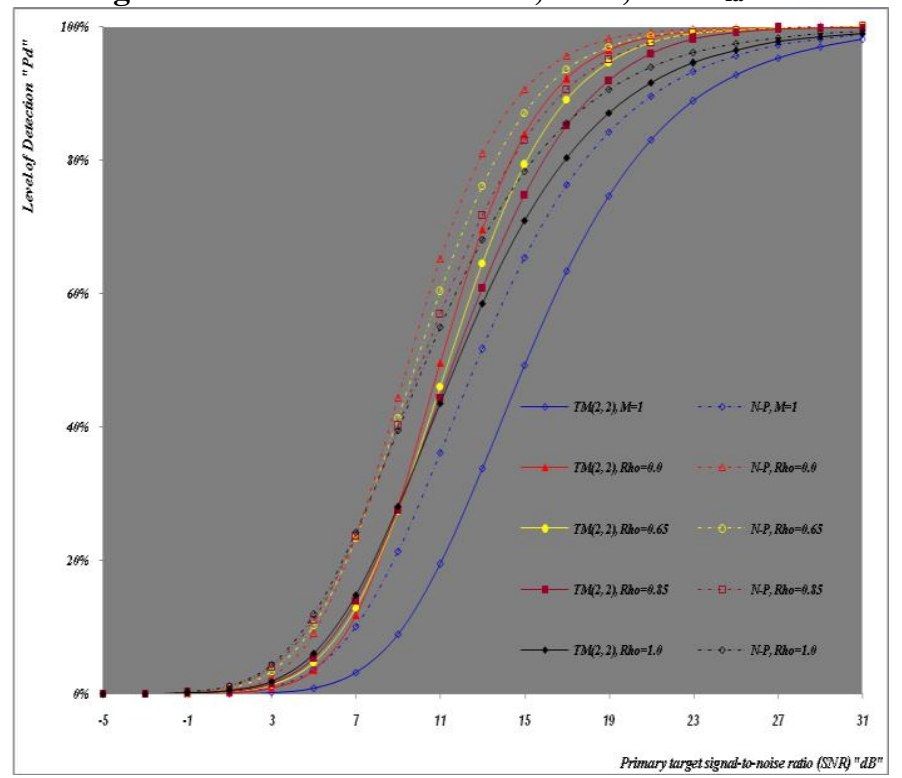

Fig.9 Multi-pulse multi-target detection performance of $\operatorname{TM}(2,2)-C F A R$ scheme for $\chi^{2}$ fluctuating targets with two-degrees of freedom when $\mathrm{N}=24, \mathrm{M}=2, \mathrm{r}_{1}=\mathrm{r}_{2}=1$, INR=SNR, and $P_{\text {fa }}=\mathbf{1 0}^{-6}$

A big insight into the variation of the curves of these plots demonstrates that they follow the same behavior of their corresponding ones in the case of the OS detector with minor enhancement either the operating environment is homogeneous or contaminated with extraneous target returns. From the displayed results of this group, it is noted that the novel methodology (LF) has very excellent detection performance in the absence (homogeneous) as well as in the presence (inhomogeneous) of outlying target returns.

To better examine the superiority of LF strategy, the required signal strength to reply a given level of 
detection for a pre-assigned rate of false alarm is employed as a metric to compare its behavior with the original procedures along with the N-P algorithm when the CFAR circuit is provided with a non-coherent integrator of M-pulses and the background is homogeneous. This is the scope of the next category which includes Fig.(10).

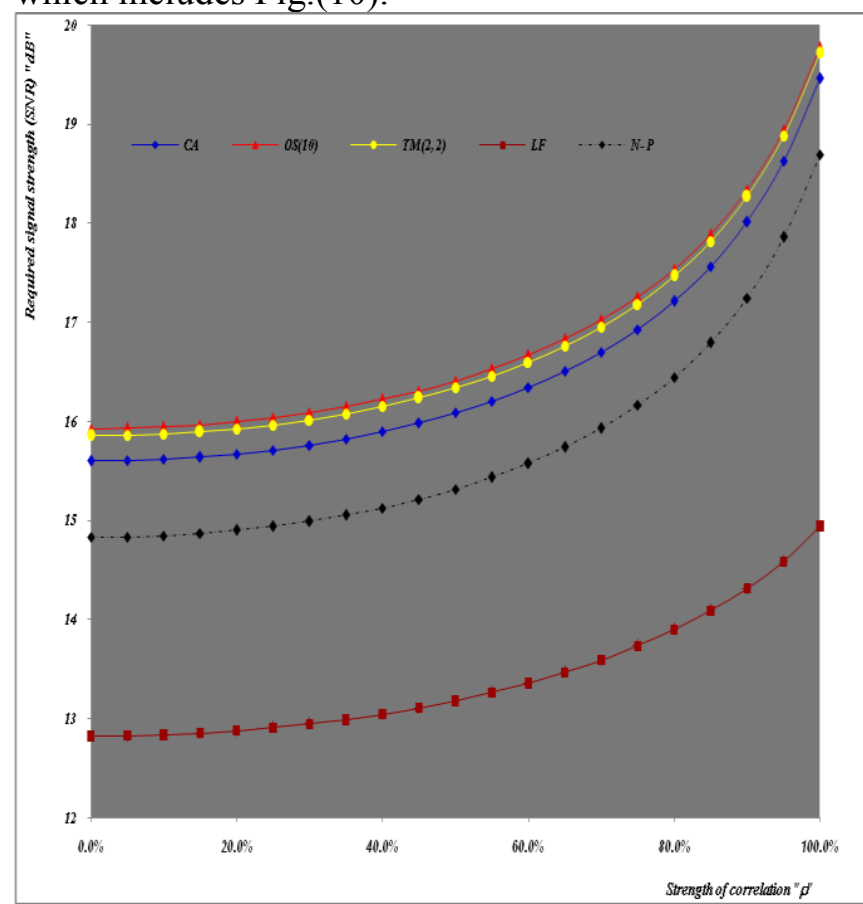

Fig.10 Multi-pulse signal strength needed to achieve a homogeneous detection level of $90 \%$ of CFAR schemes for $\chi^{2}$-fluctuating targets with two-degrees of freedom when $N=24, M=2$, and $P_{f a}=10^{-6}$

This plot is devoted to show the primary signal strength required to attain a detection level of $90 \%$ given that the radar receiver integrates two consecutive sweeps $(M=2)$ and it is designed to guarantee a false alarm rate of $10^{-6}$. To be taken as a reference of comparison, the same characteristic of the N-P detector is joined to the elements of the current scene. A big insight into the variation of the curves of this figure shows that as the correlation among the target returns becomes strengthen, more powered signal is required to reply the demanded level of detection. Additionally, the LF methodology needs the minimum signal power to satisfy the requested level of detection. Moreover, there is a large gap between the LF required signal strength to satisfy $90 \%$ level of detection and that needed by N-P processor to attain the same level of detection. This difference is slowly increasing as the correlation among the target returns augments. This behavior confirms the previously noted remarks about the detection performance of the LF structure. Moreover, the conventional CA processor calls for more signal strength than the optimum scheme, the traditional $\operatorname{TM}(2,2)$ detector needs more signal power than the CA variant to verify the detection level, and the standard OS demands the highest signal strength to accomplish the prerequisite level of detection. This sequence of the considered processors in satisfying the pre-assigned operating point $\left(\mathrm{P}_{\mathrm{d}}=0.90, \mathrm{P}_{\mathrm{fa}}=10^{-6}\right)$ exhibits that the LF methodology needs the weakest signal strength, whilst the standard OS demands the strongest signal power to verify the given operating point. This means that the LF model becomes more attractable than the N-P scheme in detecting the two-degrees of freedom partially-correlated $\chi^{2}$-target when the returned signal strength is modest.

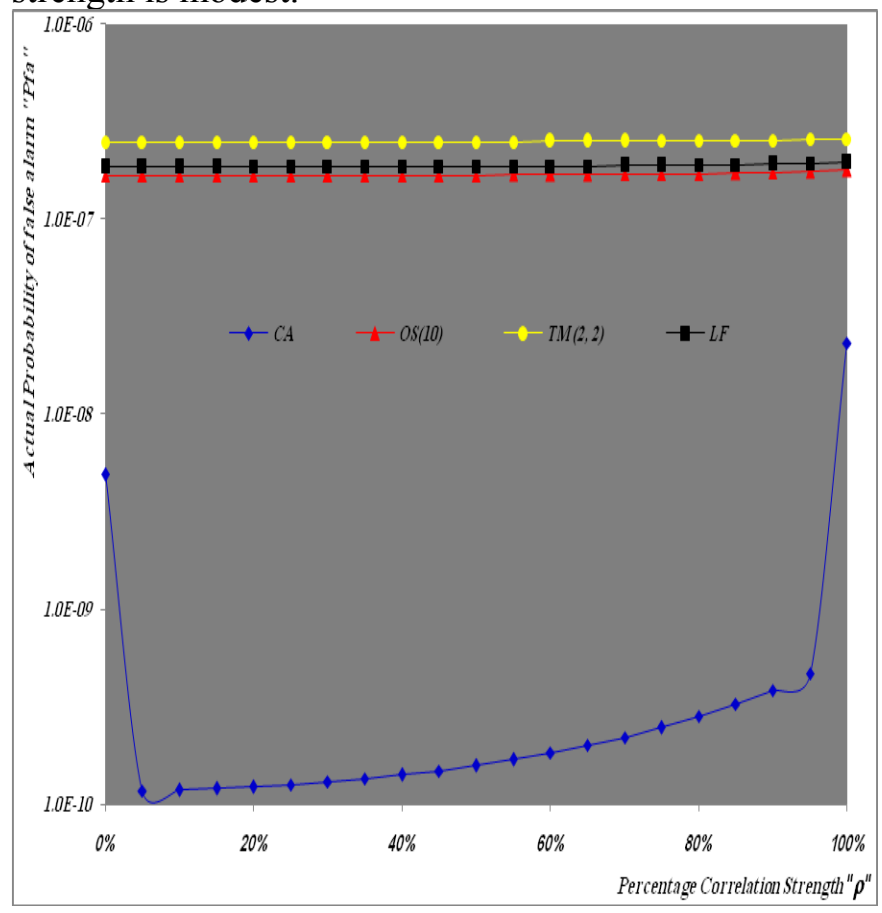

Fig.11 Actual false alarm probability of multi-pulse operation of CFAR detectors for $\chi^{2}$-fluctuating targets with two-degrees of freedom when $\mathrm{N}=24, \mathrm{M}=2, \mathrm{r}_{1}=\mathrm{r}_{2}=1$, INR=10dB, and design $P_{\mathrm{fa}_{\mathrm{a}}}=\mathbf{1 0}^{-\mathbf{6}}$

Finally, let us examine the effects of existence fallacious target returns amongst the candidates of the estimating cells on the ability of the underlined schemes to preserve the prerequisite rate of false alarm. In Fig.(11), we present some numerical results that depict the false alarm performance of the examined CFAR detectors when the sample set has some of its candidates that are contaminated with outlying target returns of equal strength. This plot shows the variation of the false alarm rate as a function of the strength of correlation among the target returns that have a signal strength of $10 \mathrm{~dB}$ when only one sample in each reference subset is doped with spurious target return $\left(r_{1}=r_{2}=1\right)$ and the CFAR circuit is provided by a noncoherent integration of two consecutive sweeps $(\mathrm{M}=2)$. The exhibited results demonstrate that the LF methodology, the conventional OS, and TM schemes are the only ones that are capable of holding the rate of false alarm fixed near its designed value; irrespective to the correlation of the interferer's returns, whilst the CA processor fails to satisfy this requirement. 


\section{Conclusions}

A comparative study associated with the detection of partially-correlated targets of the LF scenario against CA, OS, and TM mechanisms is done. This paper investigates the detailed analysis of the performance of the new methodology of CFAR detection in the absence as well as in the presence of interferers in the case where the target of interest and the outlying ones fluctuate and follow $\chi^{2}$-distribution with two-degrees of freedom in their fluctuation. Special interest is given to the partially-correlated target returns. Closed form expressions for the detection and false alarm probabilities are derived and the performance of the proposed strategy along with its fundamental versions is analytically evaluated and numerically simulated. The results show that the new technique achieves a satisfactory performance and performs well under general conditions. The simulation results reveal that the LF strategy outweighs, in its detection performance, the standard N-P processor which is taken as a reference in any new implementation of the CFAR world. Additionally, the displayed results exhibit that there is an evident enhancement in the processor performance when the radar receiver is provided by a non-coherent integrator. Moreover, the attainable gain through the technique of the non-coherent integration is maximum when two successive pulses are integrated, whereas it decreases as this number increases. In this regard, the LF formalism can detect radar targets that cannot be detected by the CA procedure which is considered as the king of the CFAR processors. More specifically, it is shown that the LF version can offer unique merits in terms of detection performance improvement in comparison with the state-of-the-art CFAR scenarios. In heterogeneous situation, on the other hand, the novel model possesses a detection behavior which is closed to that of the N-P scheme and can surpass it for stronger target returns.

In summary, the theoretical analysis along with the simulation results demonstrate that the LF technique has better detection performance either the operating environment is free of or contaminated with some interferer's returns. It is also capable of maintaining the false alarm rate fixed en face of the existence of such returns among the contents of the estimating cells. In addition, it is noticed that increasing the number of integrated pulses enhances the processor performance due to the signal gain obtained by integration and the CFAR processor performance degrades as the correlation strength, among target returns, increases and the partially-correlated cases are always enclosed by Swerling fluctuation models (SWII \& SWI). Finally, the LF methodology as well as the conventional TM and OS procedures are the only ones that have the ability of detecting the target of interest in multi-target situations.

Another target fluctuation models can be treated in the future to see the reaction of LF strategy a

\section{References}

[1] W. Q. Wang, "Radar Systems: Technology, Principals and Applications", Nova Science Publishers, Inc, 2013.

[2] El Mashade, M. B., "M-sweeps detection analysis of cell-averaging CFAR processors in multiple target situations", IEE Radar, Sonar Navig., Vol.141, No.2, (April 1994), pp. 103-108.

[3] El Mashade, M. B., "Monopulse detection analysis of the trimmed mean CFAR processor in nonhomogeneous situations", IEE Proc. Radar, Sonar Navig., Vol. 143, No. 2, pp.87-94, April 1996

[4] J. R. Machado-Fernández, N. Mojena-Hernández, J. Bacallao-Vidal, "Evaluation of CFAR detectors performance", ITECKNE Vol. 14, No. 2, December 2017, pp. $170-178$.

[5] El Mashade, M. B., "Partially correlated sweeps detection analysis of mean-level detector with and without censoring in nonideal background conditions", Int. J. Electron. Commun. (AEÜ), Vol.53, No.1, (Feb. 1999), pp. 33-44

[6] T. Laroussi and M. Barkat, "A Performance Comparison of two Time Diversity Systems using CMLD-CFAR Detection for Partially Correlated ChiSquare Targets and Multiple Target Situations", 14th European Signal Processing Conference (EUSIPCO 2006), Florence, Italy, September 4-8, 2006.

[7] El Mashade, M. B., "Analysis of Cell-Averaging Based Detectors for $\chi^{2}$ Fluctuating Targets in Multitarget Environments", Journal of Electronics (China), Vol.23, No.6, pp. 853-863, 2006.

[8] A. Farrouki and M. Barkat, "Automatic censoring CFAR detector based on ordered data variability for nonhomogeneous environments", IEE Proc.-Radar Sonar Navig., Vol. 152, No. 1, pp. 43-51, February 2005.

[9] El Mashade, M. B., "Performance Analysis of OS Structure of CFAR Detectors in Fluctuating Target Environments", Progress In Electromagnetics Research C, Vol.2, pp. 127-158, 2008.

[10] El Mashade, M. B., "Postdetection integration analysis of the excision CFAR radar target detection technique in homogeneous and nonhomogeneous environments", Signal Processing "ELSEVIER", Vol.81, (Aug. 2001), pp. 2267-2284.

[11] El Mashade, M. B., "Partially-Correlated $\chi^{2}$ Targets Detection Analysis of GTM-Adaptive Processor in the Presence of Outliers", I.J. Image, Graphics and Signal Processing, Vol. 7, No. 12, pp. 70-90, Dec. 2014.

[12] El Mashade, M. B., "Adaptive Detection Enhancement of Partially-Correlated $\chi^{2}$ Targets in an 
Environment of Saturated Interference", Recent Advances in Electrical \& Electronic Engineering, Vol. 9, No. 2, pp.1-21, 2016.

[13] El Mashade, M. B., "Heterogeneous Performance Analysis of the New Model of CFAR Detectors for Partially-Correlated $\chi^{2}$-Targets", Journal of Systems Engineering and Electronics, Vol. 29, No. 1, February 2018, pp. $1-9$.

[14] El Mashade, M. B., "Performance superiority of CA_TM model over N-P algorithm in detecting $\chi^{2}$ fluctuating targets with four-degrees of freedom", Int. J. Systems, Control and Communications, Vol. 11, No. 1, pp. 92-118, 2020.

[15] A. R. Elias-Fustfc, A. Broquetas-Ibars, J. P. Antequera, J.C. M. Yuste, "CFAR Data Fusion Center with inhomogeneous Receivers", IEEE Transactions on Aerospace and Electronic Systems, Vol. 28, No.1, January 1992, pp.276-285.

[16] Santos Lopez-Estrada, René Cumplido, "Fusion center with neural network for target detection in background clutter", Proceedings of the Sixth Mexican International Conference on Computer Science
(ENC'05), 2005.

[17] Dejan Ivković, Milenko Andrić and Bojan Zrnić, "Fusion CFAR detector in receiver of the software defined radar", 14th International Radar Symposium (IRS), Dresden, DE, Jun 19-21, 2013.

[18] Wenguang Wang, Yu Ji and Xiaoxia Lin, "A Novel Fusion-Based Ship Detection Method from Pol-SAR Images", Sensors 2015, 15, 25072-25089.

[19] Dejan Ivković, Milenko Andrić and Bojan Zrnić, "Detection of Very Close Targets by Fusion CFAR Detectors", Scientific Technical Review, Vol. 66, No. 3, pp.50-57, 2016.

[20] El Mashade, M. B., "Inhomogeneous Performance Evaluation of a New Methodology for Fluctuating Target Adaptive Detection", Progress In Electromagnetics Research C, Vol. 107, 273-286, 2021.

[21] El Mashade, M. B., "M-Sweeps multi-target analysis of new category of adaptive schemes for detecting $\chi^{2}$-fluctuating targets", Journal of Information and Telecommunication, Vol. 4, No. 3, 314-345, 2020.

\section{Creative Commons Attribution License 4.0 (Attribution 4.0 International, CC BY 4.0)}

This article is published under the terms of the Creative Commons Attribution License 4.0

https://creativecommons.org/licenses/by/4.0/deed.en_US 